\title{
Electrically conductive geomembrane enhances construction quality assurance at post installation
}

\author{
Hoeboon $\mathrm{Ng}^{\text {i) }}$ and Yongya Zheng ${ }^{\text {ii) }}$ \\ i) Senior Technical Manager, GSE Lining Technology Co., Ltd., 555 Rasa Tower 26th Floor, Phaholyothin Road Soi 19, Chatuchak, \\ Bangkok, 10900 Thailand. \\ ii) Technical Manager, GSE Environmental Lining Technology (Suzhou) Co. Ltd., Shanghai Branch Company, Room 1807-1808, Floor \\ 18 Shanghai Trade Center, No.800 Quyang Road, Hongkou District, Shanghai 200437 China.
}

\begin{abstract}
Construction Quality Assurance (CQA) plays an important role in producing a quality containment system. Electrical liner integrity surveys are non-destructive CQA tests that help locating leaks and reducing defects at post-installation of the liner systems and even after geomembranes are covered with soil and water. A new and innovative geomembrane, GSE Leak Location Liner incorporates a thin layer of electrically conductive layer on the bottom surface of an insulating geomembrane that allows it to be $100 \%$ sparked tested for defects. Liner integrity surveys on electrically conductive geomembranes have been tested and verified as the most effective and accurate candidate in locating leaks in installed geomembranes after cover soil installation. Based on large scale field tests and commercial scale tests that have been performed by third party surveyors, the leak surveys demonstrate that an electrically conductive geomembrane can significantly increase the quality of electrical liner integrity surveys by improving both speed, accuracy and expanding detection limits. It also allows the use of liner integrity surveys in applications that were previously impossible such as multiple-layer systems, on side slopes and in situation where wrinkling has occurred in the liner.
\end{abstract}

Keywords: conductive geomembrane, CQA, liner integrity survey, spark test, leak detection.

\section{INTRODUCTION}

A proper geomembrane installation with associated construction quality assurance (CQA) is always crucial to the long term performance of liner system. To ensure the sealing integrity of geomembrane installation, a series of the quality assurance and quality control program as well as field monitoring and leak tests are usually required in order to serve as verification during and after construction. Electrical liner integrity survey (ELIS) or also known as leak location (detection) survey is currently the most effective and practical means of locating leaks in installed geomembranes, particularly at post-installation after cover soil placement. Electrical liner integrity surveys as detailed in ASTM D7007 are performed using the concept that if there is a sufficiently conductive medium both above (cover material) and below (subgrade soil or a conductive geomembrane layer) an insulating barrier (geomembrane) and each of these conductive mediums are oppositely electrically charged, electric current will flow towards and through any location where the integrity of the insulating barrier has been compromised. This current flow can then be detected using equipment that measures voltage drop between two measuring points. By taking these voltage drop measurements in a predetermined grid on the cover soil, holes can be pinpointed by finding locations where the current reverses direction.

The key factors in being able to successfully perform an electrical liner integrity survey as described above are that there must be a sufficiently conductive medium both above and below the geomembrane liner, and that these conductive mediums must be in intimate contact with the geomembrane so that an electrical connection can be made through any holes in the geomembrane liner. For single-lined system using nonconductive traditional liner, an electrical liner integrity survey can only be performed successfully provided that the liner is in intimate contact with the subgrade soil that maintains sufficient natural moisture content. However, in most of the applications, that is not always a guaranteed condition to perform an electrical liner integrity survey on traditional geomembrane liner. In addition, covered ELIS cannot be performed easily on a double lined pond where the primary geomembrane liner is separated from the conductive subgrade by a geonet and a secondary liner. Similarly, even in a single lined pond, a hole that is located at the peak of a wrinkle in the liner is unlikely to be detected by ELIS on the traditional liner because 
the electrical connection is broken by the lack of intimate contact between the hole and the subgrade soil. In addition, in cases where there is a dry subgrade or encapsulated geosynthetics clay liner (GCL), the material beneath the nonconductive traditional liner may not be sufficiently conductive to perform the leak survey (Peggs 2007). Although hydrating encapsulated GCL has been suggested, it has not been embraced by the industry and GCL desiccation is still an unresolved issue when performing an electrical leak detection survey on traditional liner (Beck et al. 2013).

However, when using electrically conductive geomembrane liner, the conductive layer in the geomembrane will serve as the conductive medium at the base of geomembrane and the aforementioned issues are no longer the concerns. In most cases cover material such as water and soil will still be used as the conductive medium above the insulating barrier. In this way, an electrically conductive geomembrane will allow electrical leak detection surveys to be performed effectively in applications that were previously not possible when using traditional geomembrane liner. A series of tests including test pads, large scale test and commercial scale tests utilizing electrically conductive liners that have been performed by third party surveyors shall be discussed in the following sections.

\section{ELECTRICALLY CONDUCTIVE GEOMEMBRANE}

Historically, electrically conductive liner is a specialty polyethylene geomembrane that incorporates an electrically conductive layer which can be utilized for post-installation surface inspection, i.e., spark testing as per ASTM D7240. Spark testing using holiday detector was originally developed to inspect coatings on steel pipe. To perform spark testing, a high electrical potential (voltage) of negative polarity (ground) is applied to the metal pipe to be tested. An electrode (wand and brush) of opposite (positive) polarity is then passed over the coating. Any voids in the coating will establish continuity and allow a spark to pass between the metal pipe and the electrode (wand and brush). Geomembrane manufacturers use this technology for electrically spark testing of synthetic polyethylene geomembrane for defects and pin holes during the manufacturing process (Youngblood et al. 2009). Today, this proven technology has been adopted in the field. With the development of a specialty coextruded geomembrane having an integral outer layer of conductive material, an electrically conductive geomembrane can be $100 \%$ electrically spark tested in the field to inspect for post-installation mechanical damage. Today the specialty geomembrane also enables leak detection surveys to be effectively conducted over entire geomembrane surface even with shallow cover soils and water. Figure 1 shows a typical cross section of an electrically Conductive White geomembrane with an intimate conductive layer at the bottom surface of the liner.

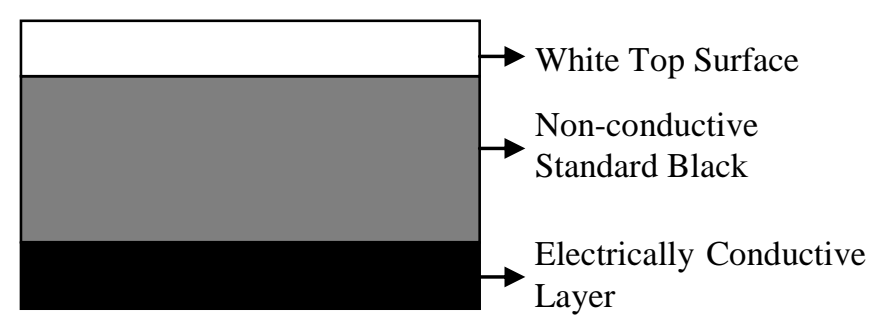

Fig. 1. Cross-section of electrically conductive geomembrane (GSE Leak Location Liner).

\section{FIELD TESTS}

\subsection{Preliminary testing}

Prior to full scale implementation, preliminary testing and investigations were performed on small scale test pads with the objective to confirm the application of electrically conductive liner for use in liner integrity surveys in an easily controllable environment that would eliminate many of the variables experienced in larger projects. As shown in Figure 2, the preliminary test pads were two $6 \mathrm{~m} \times 12 \mathrm{~m} \times 0.3 \mathrm{~m}$ above ground framed pads, one for water covered testing, and the other for soil covered testing. The frames were built on top of a large area of non-conductive textured liner which served to isolate the conductive liner from any influence by contact with the sub-grade. Additionally, in both areas geonet was also placed below the conductive liner to aid in the drainage of water from under the liner which may have had an effect on the results. To further simplify the tests and avoid any potential complications brought by welding multiple sheets, a single seamless sheet of electrically conductive geomembrane was used in each test pads.

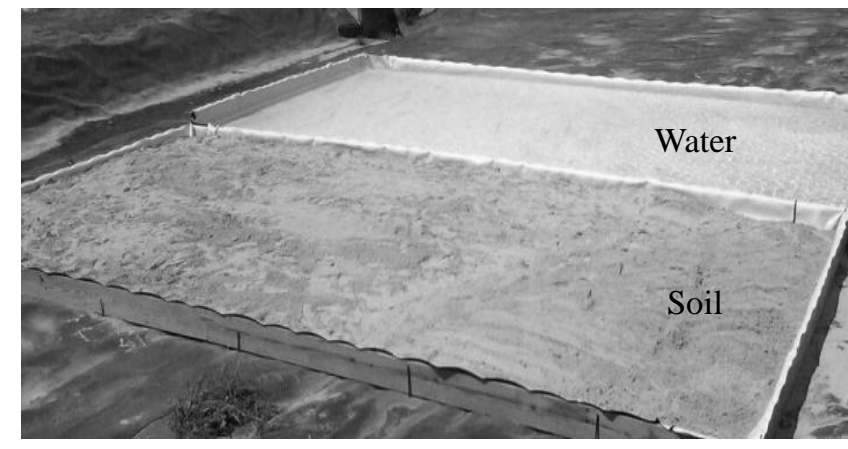

Fig. 2. Small test pads with water and soil covered.

After covering the two test pads with water and sand respectively, a traditional leak detection survey was conducted in each test pads in accordance with ASTM D7007. The surveys were first performed on the areas as constructed to verify that there were no existing holes in the liner. Following the initial survey, a small $2.5 \mathrm{~mm}$ hole was cut in each of the test areas and a 
second survey was conducted. The only additional modification to the traditional electrical leak detection survey procedure that was used in the second survey on these same test pads was connecting the ground of the power supply directly to the bottom conductive layer of the conductive geomembrane instead of the subgrade soil. This modification is required in this application because the electrically conductive liner does not make intimate contact with the subgrade soil for the reason that the electrically conductive liner is underlain by the geonet and lower geomembrane.

Results from this first survey were successful and holes that were detected were $2.5 \mathrm{~mm}$ in diameter, and with a detectable signal from approximately 3 meters away (Ramsey et al. 2012). Following with this, the next test was to introduce multiple geomembrane sheets into a larger test area. A larger test pad, $17 \mathrm{~m} \times 37 \mathrm{~m} \mathrm{x}$ $0.3 \mathrm{~m}$ was constructed and arranged as shown in Figures 3 and 4, which has included both fusion welded panel seams and extrusion welded patches at the intersections with the cross seam. In order to electrically connect adjacent panels inverted segments of conductive liner were placed beneath the seams prior to welding. The panel connector configuration shown was used so that the longest current path possible from the hole to the ground point could be tested, simulating a similar circuit over a greater distance in a smaller area, in this case a ground path distance of almost 33 meters. The containment was then filled with water and a leak survey was performed.

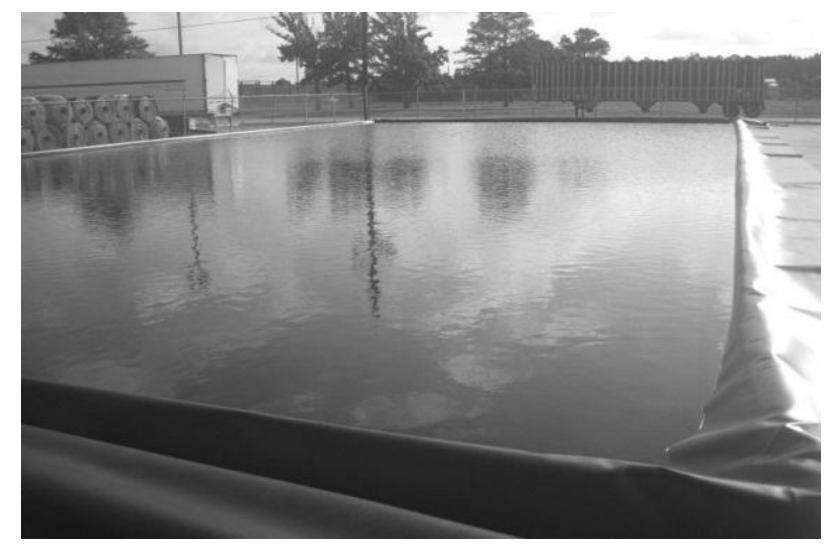

Fig. 3. Photo of test pad 3.

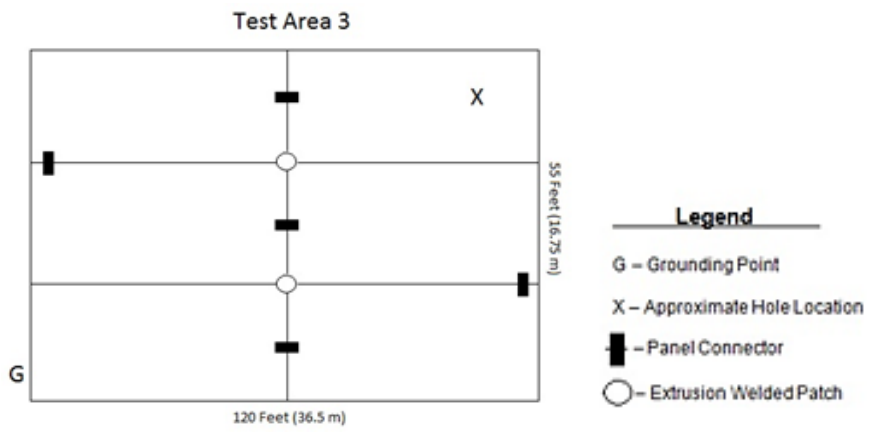

Fig. 4. Schematic of test pad 3.
The electrical liner integrity (ELI) survey on the third test pad was also successful. $2.5 \mathrm{~mm}$ diameter holes were easily located 3 meters away from the hole and showed no signal interference from the seams. Similar to the earlier tests the entire bottom surface of the geomembrane was isolated from the subgrade. In order to further demonstrate the benefits of conductive liner in leak detection surveys a hole was made on top of a wrinkle in the liner. This hole was also detected just as easily as in all previous surveys. To further confirm these results, a third party leak detection surveyor based in Texas, USA was invited to perform an electrical leak detection survey on the site using equipment that was fundamentally similar to the equipment previously used but was designed and used slightly differently. The results of this survey were equally successful and all of the holes were located (Ramsey et al. 2012).

\subsection{Large scale test}

A larger scale implementation and performance testing was conducted on a $30 \mathrm{~m} \times 76 \mathrm{~m}$ test site. Subgrade at the site was compacted soil. In some areas where sharp rocks were present, was covered with a $5 \mathrm{~cm}$ layer of sand. The first layer of geomembrane was a $0.5 \mathrm{~mm}$ thick geomembrane rub sheet for added protection as well as to isolate the conductive layer from the subgrade on top of which were a mix of both $1 \mathrm{~mm}$ and $1.5 \mathrm{~mm}$ thick electrically conductive liner. As per design, a 330 grams per square meter (10ozs. per square yard) geotextile was also laid on the liner to protect it from rocks in the $0.5 \mathrm{~m}$ cover material that was acquired from natural soil near the site.

Upon completion of site construction an electrical leak detection survey was performed by a third party surveyor based in Florida, USA. Measurements were taken at approximately $1.5 \mathrm{~m}$ intervals throughout the test area. Over the course of the survey four leaks were found, approximately $5 \mathrm{~cm}$ in length, where rocks from the cover soil had been forced down through the textile and liner. Additionally a $3 \mathrm{~mm}$ hole was also located that had been created intentionally by hand before the survey. Signal strength was primarily influenced by the size of the hole, as larger holes had a much more clear and easy to pinpoint signal however it is also suspected that inconsistencies in cover soil moisture content may also have had an effect on signal strength and clarity.

\subsection{Project performance tests}

The first project is a commercial evaluation of the electrically conductive geomembrane liner system occurred at a private surface water impoundment located at Knox County, Ohio, northeast of the city of Columbus, USA. The site was 1.6 hectares in size with an irregular shape and approximately $3 \mathrm{H}$ : $1 \mathrm{~V}$ side slopes and a maximum depth of 7.5 meters. The profile of the lining system was as follows (subgrade to 
surface): subgrade soils (clay type with shale inclusion), $0.5 \mathrm{~mm}$ thick High Density Polyethylene (HDPE) geomembrane, a mix of $1.0 \mathrm{~mm}$ and $1.5 \mathrm{~mm}$ white surfaced conductive HDPE geomembrane, a 330 grams per square meter nonwoven geotextile, 45 to 60 $\mathrm{cm}$ of cover soil (clay type with shale rock inclusions), and water. Figure 5 shows the test pad at this project site.

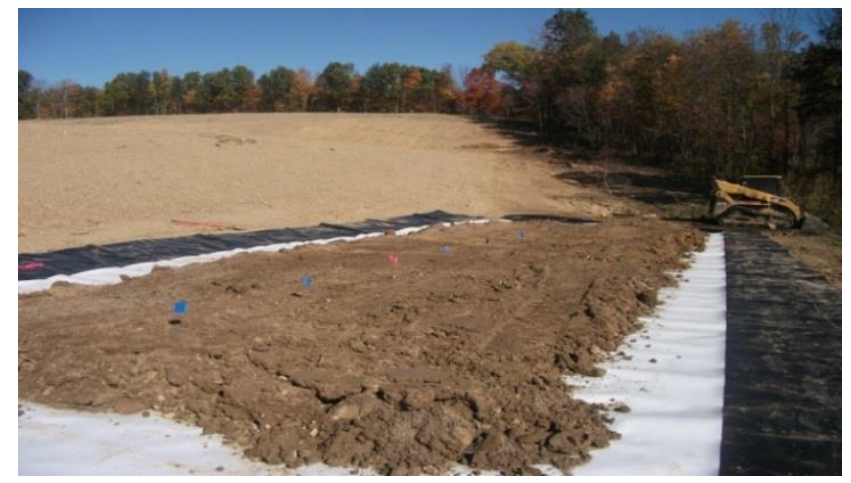

Fig. 5. Photo of test pad at Ohio site.

After installation and cover soil placement a leak detection survey was conducted by a third-party surveyor in the US. This survey was conducted through the cover soil. The $0.50 \mathrm{~mm}$ thick geomembrane rub sheet was intended to be used as a barrier to hinder electrical current flow to subgrade soil, so that any detection of leakage and liner damage in the white surfaced conductive HDPE geomembrane to be identified as a result of electrical current within the conductive geomembrane. As verification, and to help in adjusting testing protocols, a small test pad was also constructed with site specific soil that was used for equipment set-up, and to demonstrate the impact of cover soil and subgrade soil contact.

One section of approximately one half of the area of the pond had been installed with a typical welding machine and weld conditions. This technique resulted in false positive signals being generated at the flap section of the weld and prevented the accurate testing of the installation. The remainder of the site was welded using a specifically designed wedge insert for the welding equipment and performed very well. During leak testing a temporary trench was constructed to separate the two sections of the pond. Leak detection survey was successfully completed and identified the presence of 4 holes and penetrations in the geomembrane, one of which had been intentionally placed to confirm the performance of the inspection system. The necessary repairs were made and the site is currently in successful service.

The second commercial installation was completed at a construction and demolition waste landfill operation located at Mower County, Minnesota, southeast of the city of Minneapolis. The site was 1.5 hectares in size with an irregular shape and approximately $3 \mathrm{H}$ : $1 \mathrm{~V}$ side slopes. The site profile of the liner system was as follow (subgrade to surface): subgrade soils, geosynthetic clay liner, $1.5 \mathrm{~mm}$ white surfaced conductive HDPE geomembrane, geonet-geocomposite drainage product $(5 \mathrm{~mm}$ thick, double sided 130 grams per square meter geotextile), 1.2 meters of cover soil. Figure 9 shows the overview of the project site.

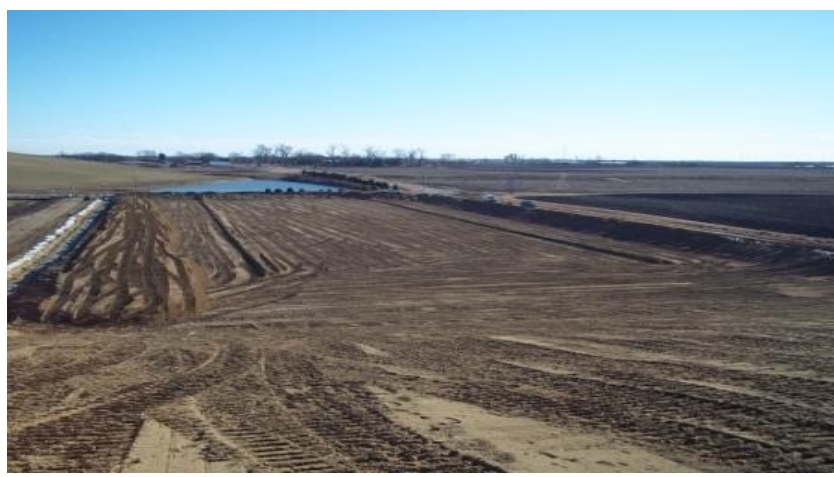

Fig. 6. Overview of Minnesota site.

After installation and cover soil placement a leak detection survey was conducted by Leak Location Services Inc. of San Antonio, Texas. This survey was conducted through the cover soil. The survey took approximately two days to complete which resulted in the discovery of a small damage in the geomembrane, a crescent shaped cut of small size (Figure 7). The necessary repair was made and the site is currently in successful service.
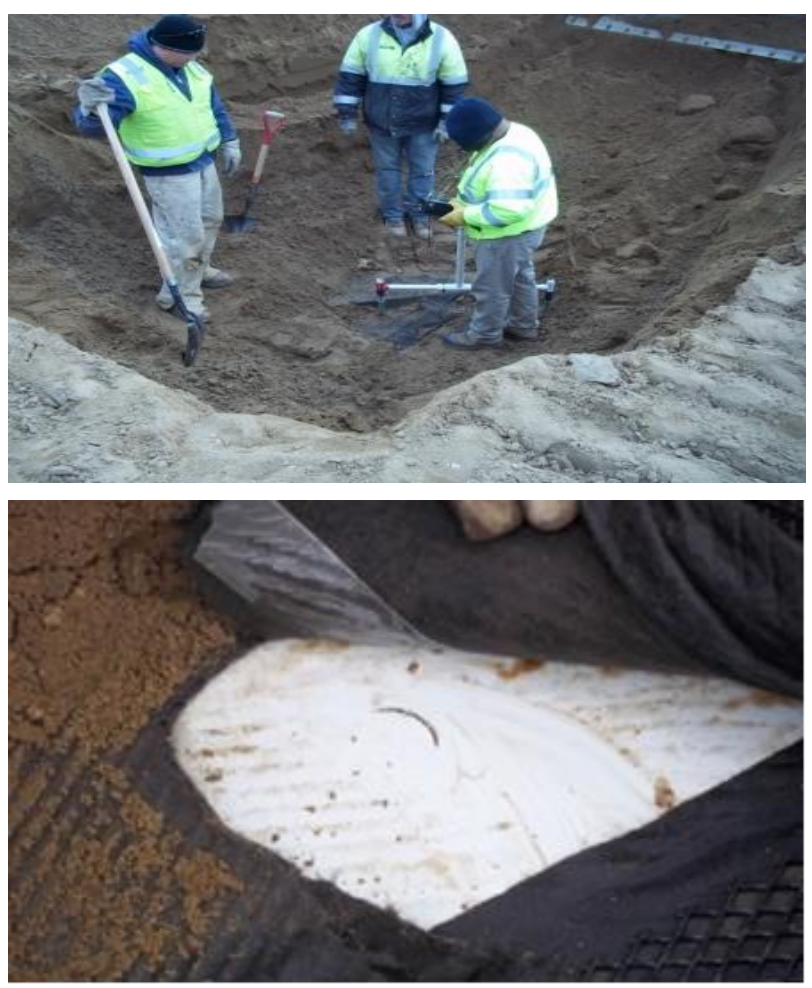

Fig. 7. Photos of defect found during covered ELI survey at Minnesota site. 


\section{CONCLUSIONS}

Covered liner integrity surveys are gaining popularity as a non-destructive CQA method for post installation leak testing. However, many conditions contribute to the reliability and performance of these types of surveys. Completion of these field performance tests has demonstrated the ability of electrically conductive geomembrane liner to be used to facilitate an electrical leak detection survey in applications that would be difficult or impossible when using traditional nonconductive liner. The recently improved electrically conductive geomembrane liner features a conductive backing specifically designed to carry sufficient electric current for leak testing to serve as the conductive medium under the geomembrane. This liner enables a leak detection survey to be completed through cover soils, and in applications where the liner is not in intimate contact with suitable subgrade, which include a double-lined pond application where the primary liner is separated from the conductive subgrade by a geonet or geocomposites and the secondary liner; the single lined pond with a geomembrane overlying GCLs and geotextiles, as well as where wrinkles occur in the liner.

The field tests also revealed that the improved electrically conductive geomembrane significantly enhances the quality of covered leak detection surveys that are used to locate holes in a liner system regardless of subsoil conductivity beneath the liner. The use of specialized welding equipment and techniques to prevent false leak indication at the seams is necessary to obtain the performance demonstrated in these field tests.

\section{REFERENCES}

1) ASTM D7007. Standard Practices for Electrical Methods for Locating Leaks in Geomembranes Covered with Water or Earth Materials. American Society for Testing and Materials, West Conshohocken, Pennsylvania, USA.

2) ASTM D7240. Standard Practice for Leak Location using Geomembranes with an Insulating Layer in Intimate Contact with a Conductive Layer via Electrical Capacitance Technique (Conductive Geomembrane Spark Test). American Society for Testing and Materials, West Conshohocken, Pennsylvania, USA.

3) Beck, A., Gallagher D., Kramer E. 2013. Leak Location Liner Performance Evaluation. Proc. of Geosynthetics 2013 Conference, Long Beach, California, USA, April 1-4, 2013.

4) Gallagher, D., Youngblood, J., Ramsey, B. 2012. New Electrically Conductive Geosynthetics in Support of Post Installation Liner Integrity Surveys. Proc. of GeoAmericas 2012, May 1-4, Lima, Peru.

5) Peggs, I. D. 2007. Liner integrity/leak-location survey: The significance of boundary conditions. Geosynthetics, February-March 2007, 34-38.

6) Ramsey, B. J., Peggs, I., Gallagher D. 2012. New Electrically Conductive Geomembrane for Post-Installation Liner Integrity Surveys. Proc. of the 5th European Geosynthetics Conference, Valencia, Spain, September 16-19, 2012.
7) Youngblood, J. \& Ward B. 2009. The Technical Background and Successful Field Experience of Spark Testing a Conductive Liner. GSE Technical Document, 2009. 